Relato de Experiência

\title{
Círculos matemáticos: uma experiência com alunos ingressantes no ensino superior no contexto da pandemia Covid-19
}

\author{
Mathematical circles: an experience with students entering higher education in the \\ context of the Covid-19 pandemic
}

\author{
Círculos matemáticos: una experiencia com estudiantes que ingresan a la educación \\ superior en el contexto de la pandemia Covid-19
}

\author{
Érika Capelato ${ }^{1}$ \\ [0000-0001-9039-6659]
}

\begin{abstract}
Resumo
Os círculos matemáticos são tradicionais em Moscou há mais de cem anos. Eles não formam um conjunto de aulas para uma disciplina, mas fomentam o encontro de alunos com professores e monitores para estudar matemática em grupos. Inspirados em seu funcionamento realizamos, junto aos alunos ingressantes do curso de Ciências Econômicas de uma universidade pública do estado de São Paulo, o projeto de ensino intitulado "círculos matemáticos da economia". O objetivo do projeto é contribuir com a melhoria da aprendizagem dos alunos que chegam à universidade com diferentes níveis de proficiência em matemática. Assim, este texto descreve e analisa a experiência da realização deste projeto no contexto da pandemia Covid-19. Para tal, lançamos mão de atividades digitais dirigidas aos participantes e das reflexões trazidas pela narrativa do monitor do projeto, através de uma produção escrita. Do ponto de vista metodológico, a discussão realizada a partir das respostas dos alunos em atividades digitais tem natureza quantitativa e mostram dados como o engajamento dos estudantes no projeto e suas percepções com respeito às atividades remotas. Já a discussão realizada em relação à narrativa tem natureza qualitativa e fundamenta-se em uma análise interpretativa, a qual nos leva a conclusão de que o convívio escolar, ainda que digital, é o ponto de partida para a construção da aprendizagem.
\end{abstract}

Palavras-chave: Círculos matemáticos. Ensino remoto. Ensino superior. Pandemia Covid-19.

\begin{abstract}
Mathematical circles have been traditional in Moscow for over a hundred years. They do not form a set of classes for a subject, but encourage the meeting of students with teachers and monitors to study mathematics in groups. Inspired by its functioning, we carried out, together with students entering the Economic Sciences course at a public university in the state of São Paulo, the teaching project entitled "Mathematical Circles of Economy". The aim of the project is to contribute to improving the learning of students who come to university with different levels of proficiency in mathematics. Thus this text describes and analyzes the experience of carrying out this project in the context of the Covid-19 pandemic. To this end, we made use of digital activities aimed at the participants and the reflections brought by the narrative of the project monitor, through written production. From a methodological point of view, the discussion based on student responses in digital activities is quantitative in nature and shows data such as student engagement in the project and their perceptions regarding remote activities. The discussion carried out in relation to the narrative, on the other hand, has a qualitative
\end{abstract}

1 erika.capelato@unesp.br, Doutora, docente, Faculdade de Ciências e Letras-UNESP, Araraquara/São Paulo/Brasil. 
nature and is based on an interpretive analysis, which leads us to the conclusion that school life, even though digital, is the starting point for the construction of learning.

Keywords: Mathematical circles. Remote teaching. University education. Covid-19 Pandemic.

\section{Resumen}

Los círculos matemáticos han sido tradicionales en Moscú durante más de cien años. No forman un conjunto de clases para una asignatura, sino que fomentan el encuentro de los alumnos con los profesores y monitores para estudiar matemáticas en grupo. Inspirándonos en su funcionamiento, realizamos, con estudiantes que ingresan a la carrera de Ciencias Económicas en una universidad pública del estado de São Paulo, el proyecto docente titulado "Círculos Matemáticos de la Economía". El objetivo del proyecto es contribuir a mejorar el aprendizaje de los estudiantes que llegan a la universidad con diferentes niveles de dominio de las matemáticas. Así, este texto describe y analiza la experiencia de llevar a cabo este proyecto en el contexto de la pandemia Covid-19. Para ello, hicimos uso de actividades digitales dirigidas a los participantes y las reflexiones que trajo la narrativa del monitor del proyecto, a través de la producción escrita. Desde un punto de vista metodológico, la discusión basada en las respuestas de los estudiantes en las actividades digitales tiene un carácter cuantitativo y muestra datos como la participación de los estudiantes en el proyecto y sus percepciones sobre las actividades remotas. La discusión realizada en relación a la narrativa, en cambio, tiene un carácter cualitativo y se fundamenta en un análisis interpretativo, lo que nos lleva a la conclusión de que la vida escolar, aunque digital, es el punto de partida para la construcción de aprendizajes.

Palabras claves: Círculos matemáticos. Enseñanza remota. Enseñanza superior. Pandemia de COVID19.

\section{Introdução}

Com a declaração feita em 11 de março de 2020 pela Organização Mundial da Saúde (OMS) caracterizando a Covid-19 como pandemia, inicia-se, em 17 de março de 2020, com a suspensão das aulas presenciais no estado de São Paulo, o período de isolamento domiciliar para os alunos da rede pública da educação básica e superior (SÃO PAULO, 2020). Os dias que seguiram a este evento foram propícios para provocar, em toda a comunidade escolar, reflexões pessoais que acreditamos serem construídas sob o contexto político, econômico, cultural e espiritual no qual o indivíduo está inserido.

Sob o aspecto individual trazido neste contexto, Peters et al (2020) coletaram narrativas de alunos em resposta à seguinte pergunta: como os alunos, conectados virtualmente, mas separados fisicamente em uma universidade internacionalizada, lidam com a ruptura provocada pela pandemia Covid-19? As narrativas apresentam, entre outros pontos, as experiências de ensino e aprendizagem online, relato das realidades e sentimentos relacionados às mudanças na vida acadêmica e discussões sobre estratégias de enfrentamento no ensino superior internacional chinês.

No que se refere a um olhar global sobre as primeiras estratégias adotadas pelos países para o ensino superior, podemos citar o trabalho de Crawford et al (2020). Nele, os autores utilizaram fontes universitárias e governamentais para fornecer um mapa das respostas do ensino superior ao período de Covid-19 em 20 países.

Ainda neste sentido, o trabalho de Arruda (2020) mostra que a excepcionalidade da situação levou, inúmeros países, a desenvolverem ações de educação remota emergencial. 
É neste cenário, de excepcionalidade, que optamos por executar, de forma remota, o projeto de ensino intitulado "círculos matemáticos da economia". Este projeto é anual e vem sendo desenvolvido junto aos alunos ingressantes do curso de graduação em Ciências Econômicas de uma universidade pública do estado de São Paulo, desde 2019.

Os círculos matemáticos são tradicionais em Moscou há mais de 100 anos e hoje inspiraram outros países a seguir esse modelo. Eles funcionam com a seguinte estrutura, são encontros semanais, não obrigatórios, feitos em horários extracurriculares, em que alunos são orientados por professores e tutores a resolverem problemas de matemática e de raciocínio lógico que nem sempre estão vinculados à estrutura curricular de uma disciplina. Assim, inspirados neste modelo, desenhamos este projeto com o objetivo de atingir os seguintes propósitos: promover integração acadêmica, contribuir para o entusiasmo em estudar matemática e melhorar a aprendizagem dos alunos que chegam ao ensino superior com diferentes níveis de proficiência em matemática.

Assim, neste trabalho fazemos o relato da experiência dos círculos matemáticos no contexto da pandemia Covid-19.

\section{Descrição da estratégia didática utilizada no ensino remoto}

Em termos didáticos, as atividades dos círculos ocorreram em 21 encontros realizados semanalmente no período extraclasse. Para os dezesseis primeiros encontros elaboramos problemas onde os participantes pudessem realizar suas tarefas de aprendizagem nos seguintes tópicos: frações; razões, proporções e regras de três; expressões algébricas; produtos notáveis; fatoração; equações, inequações e sistemas de equações do primeiro grau; equações e inequações do segundo grau e trinômios do segundo grau e cálculo de radicais. Nos cinco últimos encontros trabalhamos com problemas clássicos dos círculos matemáticos de moscou, especificamente, os problemas dos seis primeiros capítulos do livro "Um Círculo Matemático de Moscou: problemas semana a semana" do autor Sergey Dorichenko (ver Dorichenko, 2016).

No contexto do ensino remoto, criamos uma sala do Google Classroom para o projeto onde foram convidados a participar todos os alunos ingressantes no curso de Ciências Econômicas. No total, 83 alunos aceitaram o convite de serem incluídos nesta sala para participar do projeto. Os encontros síncronos das sessões aconteceram via Google Meet, sempre às segundas-feiras, com duração de 2 horas e com a presença do professor e do monitor. Para cada sessão do círculo eram postados no Google Classroom um arquivo PDF com um conjunto de problemas para serem lidos e resolvidos. Para garantir que todos os alunos tivessem acesso ao material, esta postagem era realizada com uma semana de antecedência de cada sessão síncrona. Para estas sessões que ocorriam de forma síncrona, o monitor preparava alguns slides contendo sugestões/dicas ou ainda, exemplos de solução de problemas semelhantes aos propostos no arquivo PDF da sessão. A troca de ideias e informações entre os estudantes sobre as soluções também era encorajada pelo monitor.

Em três sessões dos círculos optamos por fazer as atividades usando o Google Forms. Para cada uma destas atividade foram propostos dez problemas de múltipla escolha os quais foram elaborados utilizando os tópicos abordados em sessões anteriores. O link para o aluno ter acesso a estes problemas era disponibilizado no início da sessão, ou seja, o aluno não tinha acesso aos problemas com uma semana de antecedência. Além disso, estas sessões, foram organizadas como um modelo de avaliação classificativa, ou seja, para cada um dos dez 
problemas propostos atribuímos um ponto; assim, o score total da atividade foi dez. 0 objetivo foi evidenciar as potencialidades do instrumento e obter dados quantitativos para análise desta experiência. Previamente, informamos os participantes destas atividades que foram nomeadas, "sessão via Google Forms". Também informamos que nem o professor, nem o monitor, estariam presentes e, em nenhum momento, dissemos que estas atividades seriam "avaliativas".

Ao final de todas as sessões dos círculos colocamos no Google Classroom um questionário para que os participantes avaliassem o projeto. O questionário foi feito utilizando o Google Forms e não coletou e-mail ou nenhuma identificação dos respondentes. Na seção 3 deste texto apresentamos as questões deste questionário, bem como a análise das respostas coletadas. No que segue, apresentamos alguns dos problemas propostos em cada uma das sessões.

\subsection{Lista de problemas trabalhados em cada sessão dos círculos}

Nesta subseção vamos apresentar alguns dos problemas propostos na lista de exercícios que foi utilizada em cada sessão dos círculos. Como o número de problemas em cada lista era numeroso e, além disso, os problemas eram dotados de várias semelhanças entre si, optamos por apresentar neste texto apenas um problema de cada tipo.

Para a primeira e segunda sessão, os problemas foram propostos sob a temática de frações. Na primeira sessão optamos por exercícios que privilegiaram operações com frações como, soma, multiplicação e divisão. Além disso, trabalhamos com simplificações de expressões numéricas e frações impróprias.

Na segunda sessão foram trabalhadas situações problemas, onde foram incentivadas soluções utilizando o cálculo com frações. No Quadro 1 apresentamos alguns dos problemas destas duas primeiras sessões.

Quadro 1 - Problemas da primeira e segunda sessões

\section{Problemas da primeira sessão dos círculos}

1. Calcule a expressão $1 \frac{1}{2}+2 \frac{2}{3}$

2. Simplifique a expressão $\left(1-\frac{1}{3}\right)-\left(\frac{3}{4}+\frac{1}{8}\right)$

3. Ordene as seguintes frações em ordem crescente $\frac{1}{2}, \frac{2}{3}, \frac{1}{4}, \frac{2}{5}, \frac{5}{6}, \frac{3}{7}, \frac{5}{8}$

4. Simplifique a expressão $\left(\frac{1}{3}+\frac{3}{4}\right)\left[\left(\frac{5}{6}-\frac{1}{2}\right) \div\left(\frac{5}{6}-\frac{3}{8}\right)\right]$

Problemas da segunda sessão dos círculos

1. Qual a área aproximada do Brasil se $\frac{2}{5}$ dessa área é igual a $340.00 \mathrm{~km}^{2}$ ?

2. João tem um plano de internet de $240 \mathrm{MB}$ mensais, dos quais já gastou $50 \mathrm{MB}$. Joana, por outro lado, tem um plano de $360 \mathrm{MB}$, dos quais já usou $70 \mathrm{MB}$. Quem gastou mais (em termos relativos)?

3. Pedro tem 50 fotos armazenadas em seu smartphone, cada uma com tamanho aproximado de $140 \mathrm{~KB}$, sendo que seu celular consegue armazenar até $1 \mathrm{~GB}$ (1GB corresponde $1000 \mathrm{MB}$ e $1 \mathrm{MB}$ equivale a $1000 \mathrm{~KB}$ ). Por outro lado, Carla tem 120 fotos armazenadas, cada uma com tamanho aproximado de 130KB, e seu celular consegue armazenar até 2GB. Qual dos dois armazenou mais dados (relativamente a capacidade de armazenamento de cada um)? 
Fonte: Elaboração própria, baseado no material de Abreu; Capelato (2019).

Na terceira sessão, os problemas foram propostos sob a temática razões, proporções e regras de três. Em especial, tratamos dos conceitos de grandezas diretamente e inversamente proporcionais e suas propriedades, ou seja, uma proporção é uma igualdade entre duas razões $a / b$ e $c / d$, isto é, $\frac{a}{b}=\frac{c}{d}$.

Neste caso, dizemos que " $a$ está para $b$ assim como $c$ está para $d$ ". São propriedades aritméticas fundamentais,

$$
\frac{a}{b}=\frac{c}{d} \leftrightarrow a d=c d
$$

ou

$$
\frac{a+c}{b+d}=\frac{a}{b}=\frac{c}{d}
$$

ou mais geral,

$$
\frac{m a+n c}{m b+n d}=\frac{a}{b}=\frac{c}{d}
$$

para quaisquer $m$ e $n$.

Também abordamos a generalização destas propriedades, ou seja, uma proporção envolvendo um número arbitrário de razões: se

$$
\frac{a_{1}}{b_{1}}=\frac{a_{2}}{b_{2}}=\frac{a_{3}}{b_{3}}=\cdots=\frac{a_{k}}{b_{k}}
$$

e $m_{1}, \cdots, m_{k}$ são números reais quaisquer então

$$
\frac{m_{1} a_{1}+\cdots+m_{k} a_{k}}{m_{1} b_{1}+\cdots+m_{k} b_{k}}=\frac{a_{1}}{b_{1}}=\cdots=\frac{a_{k}}{b_{k}} .
$$

No Quadro 2 apresentamos alguns dos problemas escolhidos para a terceira sessão.

Quadro 2 - Problemas da terceira sessão

\section{Problemas da terceira sessão dos círculos}

1. Calcule $x$ e $y$ na proporção $\frac{x}{5}=\frac{y}{6}$ sabendo que $x+y=33$.

2. Divida 100 em partes proporcionais a 2 e 3.

3. Divida 46 em partes diretamente proporcionais a $\frac{1}{2}, \frac{2}{3} \mathrm{e} \frac{3}{4}$.

4. Numa fábrica, 12 funcionários produzem um determinado grupo de peças em 4 horas. Quantos funcionários com igual capacidade de trabalho, produziriam a mesma quantidade de peças em 6 horas? 
5. Uma companhia de transporte ferroviário de cargas projeta suas locomotivas de modo que:

- o rendimento (em quilômetros por litro de diesel) seja diretamente proporcional à distância entre remetente e destinatário, ou seja, quanto maior a distância, tanto maior será o rendimento;

- o rendimento é inversamente proporcional à carga transportada (como é de se esperar).

Considere uma determinada locomotiva, que transporta 10 toneladas por $50 \mathrm{~km}$ com um rendimento de $50 \mathrm{~km} / \mathrm{l}$.

(a) Qual será o rendimento da locomotiva acima ao transportar 20 toneladas por $40 \mathrm{~km}$ ?

(b) Qual deve ser a carga máxima que a locomotiva acima deve transportar num percurso de $80 \mathrm{~km}$ de modo que o rendimento seja de $10 \mathrm{~km} / \mathrm{l}$ ?

6. Laura, estudante de economia, quer ler, além de O Capital de Karl Marx e A Riqueza das Nações de Adam Smith, o livro O Capital no Século XXI de Thomas Piketty, ao longo dos próximos meses. Ela estima que, lendo um determinado número de páginas por dia, ao longo de 4 dias por semana, completaria a leitura em 6 meses. Em quanto tempo ela completaria a leitura se aumentasse $20 \%$ o número de páginas lidas por dia ao longo de 5 dias por semana?

Fonte: Elaboração própria, baseado no material de Abreu; Capelato (2019).

$\mathrm{Na}$ quarta sessão os problemas foram propostos sob a temática expressões algébricas. Assim, os problemas deste encontro foram elaborados com o objetivo de trabalhar, especificamente, as manipulações algébricas no processo de simplificação de expressões.

Na quinta sessão, os problemas foram propostos sob a temática produtos notáveis, que é um caso especial de expressões algébricas. No Quadro 3 descrevemos alguns dos problemas da quarta e quinta sessões.

Quadro 3 - Problemas da quarta e quinta sessões

\section{Problemas da quarta sessão dos círculos}

1. Simplifique a expressão algébrica

$$
\frac{2 x^{3}}{3 z} \times\left(-\frac{5 x}{4 x^{2}}\right)+\frac{5 x^{3} z^{2}}{3} \div \frac{10 x z^{3}}{9 y}
$$

2. Ache constantes $A$ e $B$ tais que

$$
\frac{5 x+1}{(x-1)(x+2)}=\frac{A}{x-1}+\frac{B}{x+2}
$$

\section{Problemas da quinta sessão dos círculos}

1. Desenvolva
(a) $\left(3 x-\frac{y}{5}\right)^{2}$
(c) $(a+b+c)^{2}$
(b) $(3 m+2 n)^{3}$
(d) $(a+b+c)^{3}$

2. Desenvolva o produto da soma pela diferença

$$
\left(3 x+\frac{y}{4}\right)\left(3 x-\frac{y}{4}\right)
$$

3. Simplifique a expressão

$$
x y(x+y)^{2}-y(x+y)^{3}
$$


Fonte: Elaboração própria, baseado no material de Abreu; Capelato (2019).

Na sexta e sétima sessões, os problemas foram propostos sob a temática fatoração, que é, num certo sentido, inverter o que fizemos na sessão anterior. Dentre as discussões desta temática, estão a fatoração dos trinômios do segundo grau:

$$
a x^{2}+b x+c
$$

A fatoração da expressão acima é baseada na identidade:

$$
(m x+p)(n x+q)=m n x^{2}+(m q+n p) x+p q .
$$

Assim, devemos achar números $m$ e $n, p$ e $q$ tais que os produtos verticais $m n$ e $p q$ (no esquema abaixo) sejam iguais, respectivamente, a $a$ e $c$, e tais que a soma do "produto cruzado" $m q+n p$ seja igual a $b$.

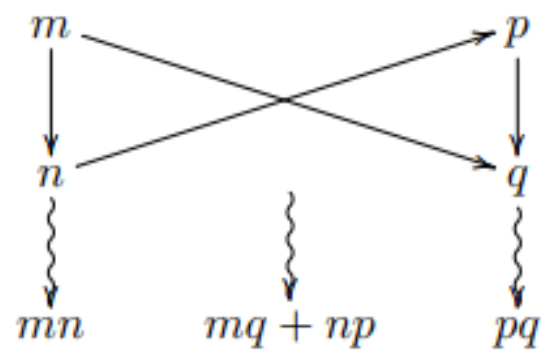

Neste caso temos

$$
a x^{2}+b x+c=(m x+p)(n x+q) .
$$

Além desta, exploramos nos problemas, a fatoração da diferença e soma de cubos, ou seja, $a^{3}-b^{3}=(a-b)\left(a^{2}+a b+b^{3}\right)$ e $a^{3}+b^{3}=(a+b)\left(a^{2}-a b+b^{3}\right)$. No Quadro 4 descrevemos alguns dos problemas da sexta e sétima sessões.

Quadro 4- Problemas da sexta e sétima sessões

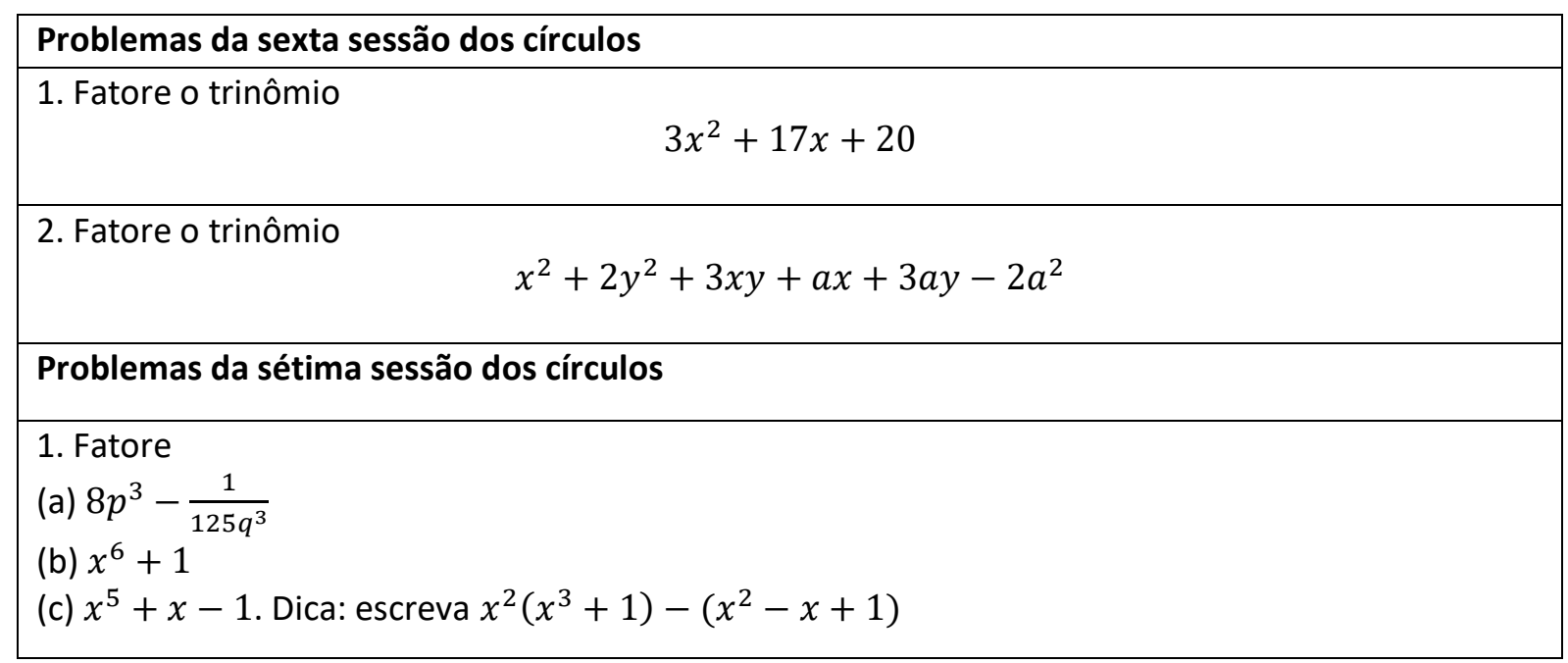

Fonte: Elaboração própria, baseado no material de Abreu; Capelato (2019).

No início da oitava e nona sessões os participantes receberam um link de acesso ao Google Forms que continha dez questões de múltipla escolha referentes aos conteúdos abordados nas sessões anteriores. Como estes problemas foram elaborados a partir de 
ínfimas variações dos problemas apresentados nos quadros anteriores, eles serão omitidos deste texto. Uma análise quantitativa dos resultados destas sessões será apresentada na seção 3 deste texto.

Na décima e décima primeira sessões os problemas foram propostos sob a temática de equações, inequações e sistemas do primeiro grau. No Quadro 5 apresentamos alguns destes problemas.

Quadro 5 - Problemas da décima e décima primeira sessões

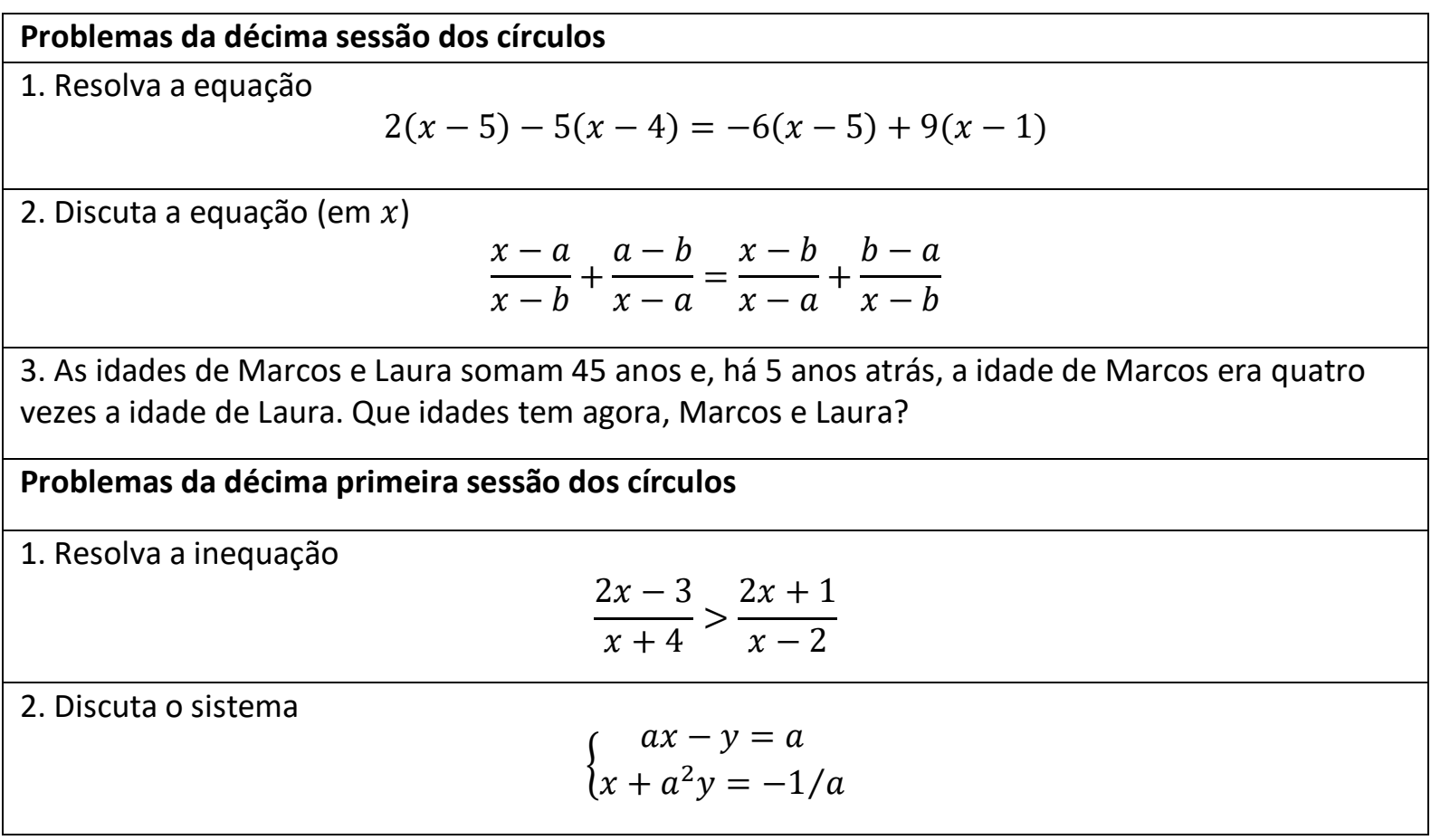

Fonte: elaboração própria, baseado no material de Abreu; Capelato (2019).

Na décima segunda e décima terceira sessões os problemas foram propostos sob a temática de equações, inequações de segundo grau e trinômios do segundo grau. Para resolução das equações de segundo grau, foi incentivado que a fatoração fosse usada, sempre que possível.

Na sequência, discutimos o seguinte resultado: dois números reais $x_{1}$ e $x_{2}$ são sempre raízes de uma equação do segundo grau, a saber, da equação $\left(x-x_{1}\right)\left(x-x_{2}\right)=0$, isto é,

$$
x^{2}-S x+P=0 \text {, }
$$

onde $S=x_{1}+x_{2}$ e $P=x_{1} x_{2}$.

Finalmente, discutimos as resoluções das equações do segundo completando quadrados. Ou seja,

$$
\begin{gathered}
a x^{2}+b x+c=0 \leftrightarrow x^{2}+\frac{b}{a} x=-\frac{c}{a} \leftrightarrow x^{2}+\frac{b}{a} x+\frac{b^{2}}{4 a^{2}}=-\frac{c}{a}+\frac{b^{2}}{4 a^{2}} \\
\leftrightarrow\left(x+\frac{b}{2 a}\right)^{2}=\frac{b^{2}-4 a c}{4 a^{2}} \leftrightarrow x+\frac{b}{2 a}= \pm \sqrt{\frac{b^{2}-4 a c}{4 a^{2}}}
\end{gathered}
$$

Logo, se $b^{2}-4 a c \geq 0$, 


$$
a x^{2}+b x+c=0 \leftrightarrow x=-\frac{b}{2 a} \pm \frac{\sqrt{b^{2}-4 a c}}{2 a}
$$

Na discussão do trinômio do segundo grau, ou seja, números reais $x$ e $y$ relacionados pela igualdade

$$
y=a x^{2}+b x+c
$$

onde $a, b$ e $c$ são constantes, discutimos a seguinte propriedade: quando $x$ percorre todos os números, $y$ atinge um extremo, máximo ou mínimo, em algum desses pontos de $x$. Para isto, basta reescrever a equação acima na forma

$$
y=a\left(x+\frac{b}{2 a}\right)^{2}-\frac{\Delta}{4 a^{2}}
$$

onde $\Delta=b^{2}-4 a c$ e observar que $\left(x+\frac{b}{2 a}\right)^{2} \geq 0$ para qualquer $x$. Assim, quando $x=$ $-b / 2 a$, a expressão

$$
-\frac{\Delta}{4 a^{2}}
$$

é o valor mínimo de $y$ se se $a>0$, e é o seu valor máximo se $a<0$.

No Quadro 6 apresentamos alguns dos problemas que foram trabalhados na décima segunda e décima terceira sessões.

Quadro 6 - Problemas da décima segunda e décima terceira sessões

\section{Problemas da décima segunda sessão dos círculos}

1. Determine $m$ de modo que as raízes da equação $x^{2}+\left(m^{2}-1\right) x-3=0$ sejam simétricas (isto é, uma raiz é o negativo da outra).

2. Discuta as equações do segundo grau

$a b\left(x^{2}+1\right)=\left(a^{2}+b^{2}\right) x$ com $a$ e $b$ constantes não ambas iguais a zero.

\section{Simplifique a expressão}

$$
1+\frac{1}{2+\frac{1}{2+\frac{1}{2+\bullet}}}
$$

A expressão acima é denominada fração continuada. O sentido dos três pontinhos é de que o padrão anterior se repete infinitamente.

\section{Problemas da décima terceira sessão dos círculos}

1. Resolva a inequação e represente sua solução geometricamente

$$
\frac{x^{2}+3 x-10}{x^{2}+4 x+3}<0
$$

2. Considere o trinômio do segundo grau $y=-x^{2}+6 x-10$. Determine o valor máximo do trinômio e em que ponto este extremo ocorre.

3. Pedro quer construir um canil em seu quintal para o seu cãozinho. De modo a aproveitar ao máximo os $10 \mathrm{~m}$ de quadril disponíveis, ele o constrói rente a uma parede. Quais as dimensões do canil de modo que sua área seja máxima? 
Fonte: Elaboração própria, baseado no material de Abreu; Capelato (2019).

$\mathrm{Na}$ décima quarta e décima quinta sessões os problemas foram propostos sob a temática cálculo de radicais. O foco dos problemas escolhidos para estas sessões foi expressões numéricas e algébricas, equações e inequações envolvendo radicais. Além disso, consideramos problemas de radicais cúbicos. No Quadro 7 apresentamos alguns dos problemas abordados nestas sessões.

Quadro 7 - Problemas da décima quarta e décima quinta sessões

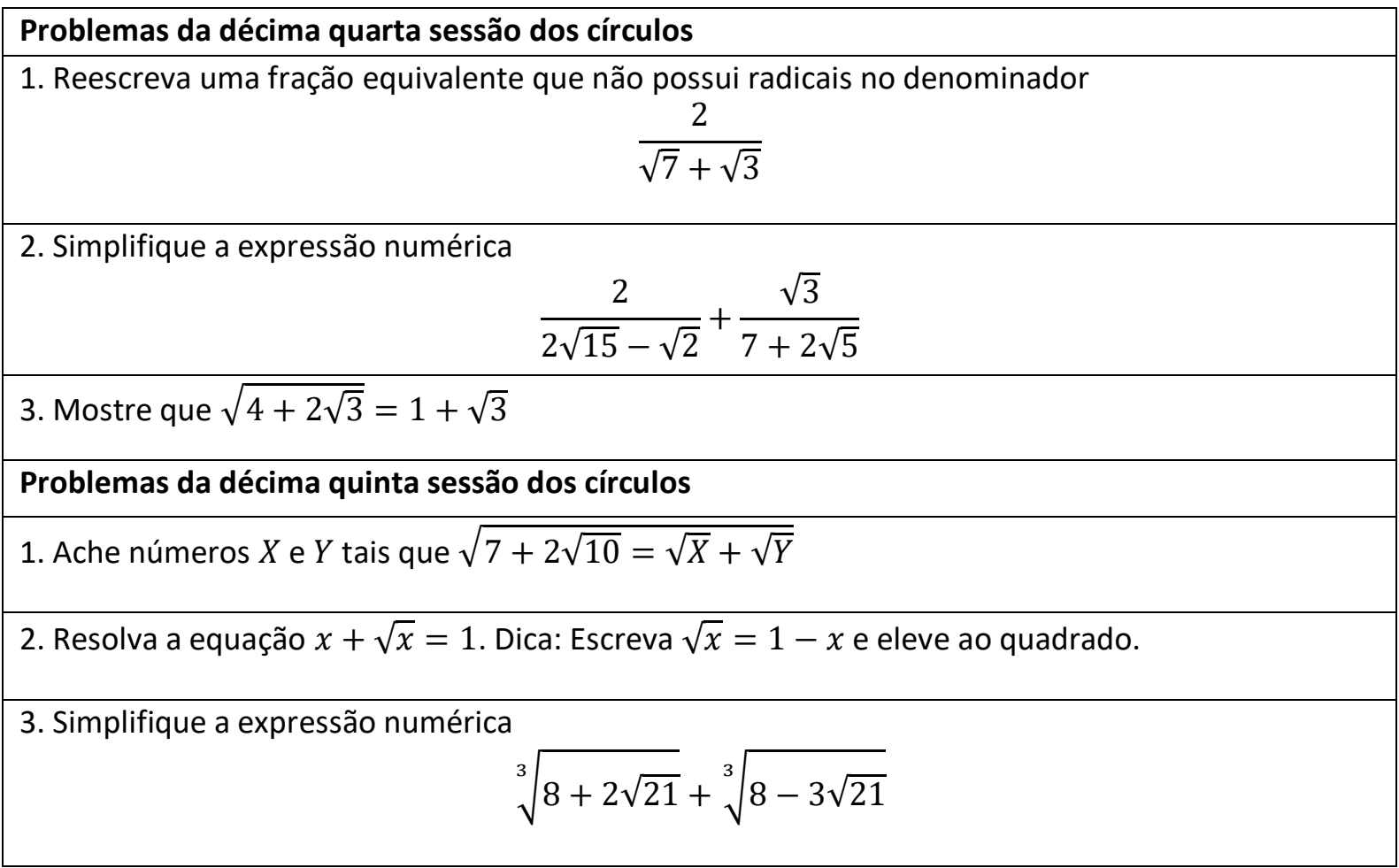

Fonte: elaboração própria, baseado no material de Abreu; Capelato (2019).

A décima sexta sessão ocorreu como a oitava e nona sessões (sessões via Google Forms). Os problemas foram elaborados a partir de ínfimas variações dos problemas apresentados nos quadros 4, 5 e 6 assim, eles serão omitidos deste texto.

Para as últimas sessões dos círculos trabalhamos com os problemas de Dorichenko (2016); por isso, não os descreveremos neste texto. Na décima sétima sessão trabalhamos com os problemas dos capítulos 0 e 1 , na décima oitava com os dos capítulos 2 e 3 . Na décima nona com os do capítulo 4; na vigésima e vigésima primeira, com os do capítulo 5 e 6, respectivamente.

\section{Resultados da experiência}

Nesta seção apresentamos os resultados da experiência que tivemos em relação a realização dos círculos matemáticos junto aos ingressantes do curso de ciências econômicas no contexto da pandemia da Covid-19.

É oportuno relatarmos que o primeiro encontro, onde explicamos os objetivos do projeto, ocorreu 50 dias após a suspensão das aulas presenciais na universidade em função da pandemia da Covid-19. Para a realização deste primeiro encontro, enviamos, pelo e-mail que o aluno havia cadastrado no ato de sua matrícula, um link para que os ingressantes 
participassem de uma reunião online realizada via Google Meet. Ao todo, 100 alunos receberam o e-mail e 83 compareceram a este primeiro encontro. Relatamos aqui que, durante estes 50 dias, nenhuma atividade remota foi iniciada com estes alunos. Assim, para esta primeira reunião as perguntas recorrentes dos alunos foram: quando voltaremos a ter aulas presenciais? Teremos aulas online?

Ainda que não conseguíssemos dar respostas a estas perguntas, os alunos se motivaram para participar semanalmente das sessões dos círculos matemáticos.

Para descrever os resultados desta experiência, primeiramente olhamos para a quantidade de alunos que participaram das sessões dos círculos, veja Figura 1. É possível observar a redução no número de participantes ao longo do período.

Outras observações bastante evidentes são: (i) o grande número de participantes nas sessões oito e nove, as quais ocorreram utilizando o Google Forms, conforme descrevemos na seção 2 deste texto e (ii) a diferença entre o número de participantes no início e término das sessões.

Figura 1 - Quantidade de alunos que participaram em cada sessão

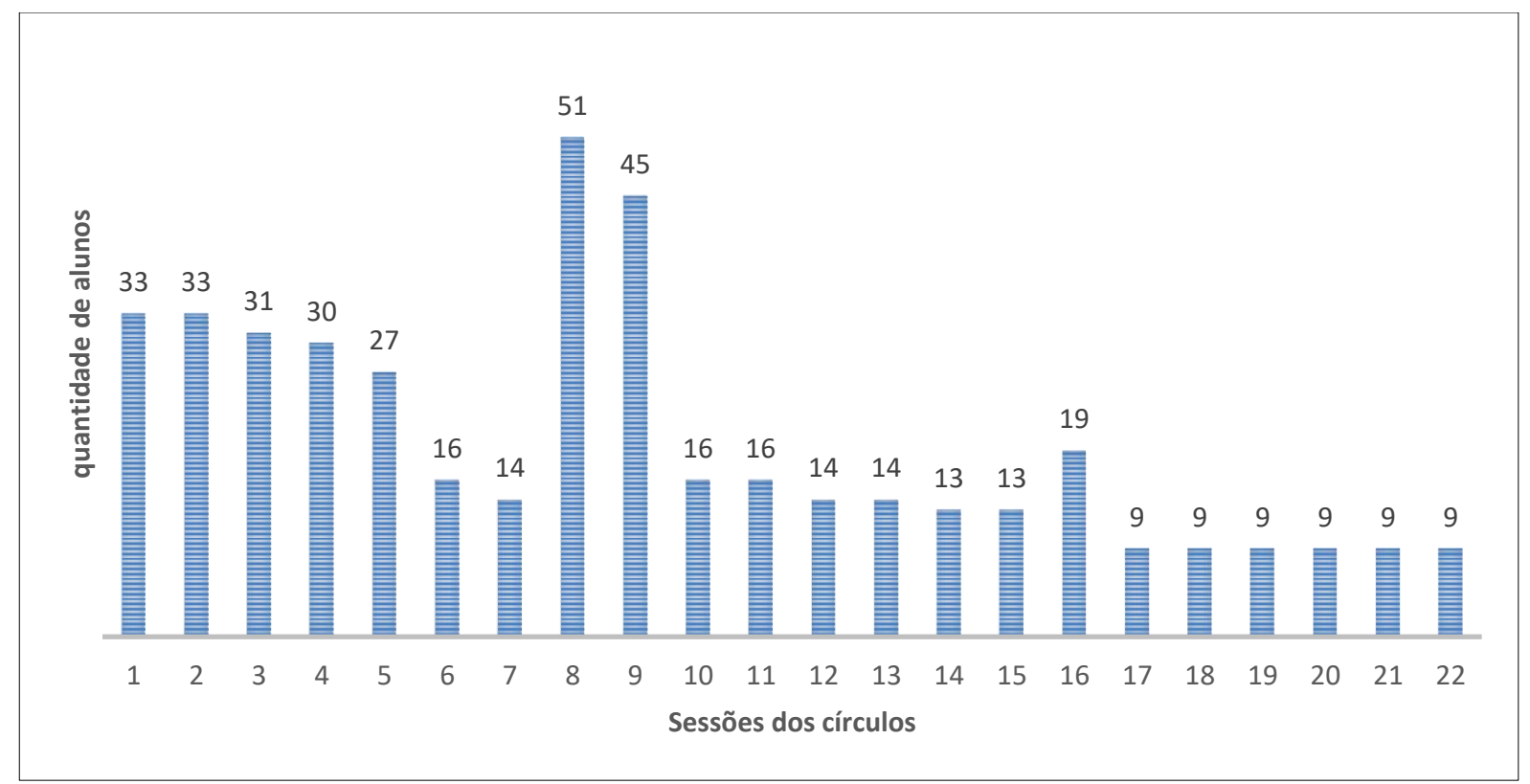

Fonte: elaboração própria com base nos dados coletados

A primeira e segunda sessões ocorreram como única atividade remota da qual os alunos ingressantes do curso de Ciências Econômicas estavam participando. Da terceira até a nona sessão, juntou-se a esta atividade, o início das atividades remotas de outras duas disciplinas obrigatórias do curso, das quais os alunos ingressantes deveriam participar. A partir da data que ocorreu a décima sessão dos círculos houve a retomada, a partir de atividades remotas, de todas as disciplinas obrigatórias do curso. O relato destes eventos não tem a pretensão de serem argumentos para justificar a participação (ou ausência) dos alunos nas sessões, mas são complementos importantes de análise de todo o contexto.

Ainda que os 83 alunos inscritos no Google Classroom do projeto não participassem das sessões dos círculos, eles permaneceram inscritos nesta plataforma (sair deste ambiente pode ser feito a qualquer momento pelo participante) até o final do projeto. Semanalmente, as listas com os problemas das sessões eram postadas neste ambiente e, dada as especificidades da plataforma, todos os alunos inscritos recebiam um e-mail de notificação 
desta postagem. Além disso, as listas de problemas ficaram disponíveis para o acesso, a qualquer momento, para todos os alunos inscritos no Google Classroom até o final deste projeto.

Da Figura 1 observamos que as sessões que ocorreram com a utilização do Google Forms (sessão 8, 9 e 16) atraíram a participação dos alunos ingressantes. Como estas atividades foram organizadas na forma teste e, a cada um dos dez problemas atribuímos um ponto, se o participante respondesse corretamente os dez problemas propostos era atribuído score 10. Na Tabela 1 trazemos o score médio obtido pelos participantes nestas atividades.

Tabela 1 - Nota média nas sessões com Google Forms

\begin{tabular}{|l|l|}
\hline Atividade & Score médio \\
\hline Oitava sessão dos círculos - Google Forms & 8,25 \\
\hline Nona sessão dos círculos - Google Forms & 9,58 \\
\hline Décima sexta sessão dos círculos - Google Forms & 8,18 \\
\hline
\end{tabular}

Fonte: Elaboração própria com base nos dados coletados

A organização de sessões utilizando o Google Forms mostrou, ainda que com poucas evidências, pois aplicamos este modelo em apenas três sessões, que o instrumento (Google Forms) atraiu a participação dos alunos, seja por ser um recurso digital; por se assemelhar a um modelo de prática avaliativa classificatória, tão presente em todos os níveis escolares e assim, presente no cotidiano dos alunos; ou ainda, por se apresentar como um desafio ao aluno, que busca "checar", de forma classificatória, seu desempenho em matemática.

Ao final de todas as sessões dos círculos, colocamos no Google Classroom do projeto um questionário para que os participantes avaliassem a experiência. O questionário foi elaborado e respondido utilizando o Google Forms. Todos os inscritos receberam a notificação e puderam acessar esta avaliação; porém, somente 14 participantes responderam o questionário. As perguntas e o percentual de respostas estão na Tabela 2.

Tabela 2 - Questionário de avaliação do projeto

\begin{tabular}{|l|l|}
\hline \multicolumn{1}{|c|}{ Perguntas } & Alternativas - Percentual de respostas \\
\hline $\begin{array}{l}\text { 1. Por favor, avalie seu nível de satisfação com o } \\
\text { material disponibilizado para as sessões. }\end{array}$ & $\begin{array}{l}\text { Muito satisfeito - } 50 \% \\
\text { Satisfeito - } 50 \% \\
\text { Muito insatisfeito - } 0 \% \\
\text { Insatisfeito - } 0 \% \\
\text { Não sei e/ou não quero opinar - 0\% }\end{array}$ \\
\hline $\begin{array}{l}\text { 2. Por favor, avalie o grau de dificuldade dos } \\
\text { conteúdos e problemas do material } \\
\text { disponibilizado em cada sessão. }\end{array}$ & Muito difícil - $0 \%$ \\
& Difícil - 71,4\% \\
& Fácil - $28,6 \%$ \\
\hline $\begin{array}{l}\text { 3. Você acha que a duração do projeto foi } \\
\text { suficiente para atender às suas expectativas? }\end{array}$ & Não sei e/ou não quero opinar - $0 \%$ \\
& Sim - $85,8 \%$ \\
\hline $\begin{array}{l}\text { 4. Você acha que a realização deste projeto de } \\
\text { forma remota o tornou menos eficaz para a sua } \\
\text { aprendizagem? }\end{array}$ & Não $-7,1 \%$ \\
\hline
\end{tabular}




\begin{tabular}{|l|l|}
\hline $\begin{array}{l}\text { 5. Considerando sua experiência completa com o } \\
\text { projeto, quais são as possibilidades de }\end{array}$ & Recomendaria - $100 \%$ \\
recomendá-lo a um aluno ingressante em Ciências & Não recomendaria - 0\% \\
Econômicas? & Não sei e/ou não quero opinar - 0\% \\
\hline
\end{tabular}

Fonte: Elaboração própria.

A análise dos dados da Tabela 2 mostra que os participantes que responderam o questionário ficaram satisfeitos ou muito satisfeitos com o material disponibilizado para cada sessão. Porém, $71,4 \%$ dos respondentes classificaram o conteúdo e os problemas contidos no material como difíceis. Quanto ao tempo de duração do projeto, $85,8 \%$ dos respondentes consideraram que foi suficiente. Finalmente, observamos que $64,3 \%$ dos respondentes indicaram que a realização do projeto de forma remota não o tornou menos eficaz para a sua aprendizagem e, todos os respondentes, recomendariam o projeto a um aluno ingressante no curso de Ciências Econômicas.

Ainda com o objetivo de analisar os resultados do projeto pedimos ao monitor, que atuou diretamente em todas as sessões dos círculos, escrevesse um relato sobre a experiência vivida com o projeto no contexto da pandemia da Covid-19. Do ponto de vista metodológico, acreditamos que a produção escrita valoriza os processos vividos e dá a oportunidade aos indivíduos de refletirem sobre todo o processo, o que enriquece sua formação acadêmica e profissional.

Abaixo está um trecho das reflexões trazidas pela narrativa do monitor:

Aquilo que já se mostrava um desafio, com as adversidades causadas pela pandemia tornouse um desafio ainda maior (...). Mesmo tendo proximidade com a tecnologia digital e com a informática, conciliar novas ferramentas com minhas primeiras experiências na monitoria foi muito difícil. Muitas dificuldades apareceram já na primeira aula, a turma estava lotada. Além de ter que lidar com problemas matemáticos, trabalhei meu emocional e o emocional dos alunos, estávamos todos ansiosos (...). Passou-se a primeira aula, e as seguintes foram sendo lentamente evacuadas, percebi uma certa falta de esperança em toda turma, não saberia julgar se por conta da pandemia e seus problemas ou em decorrência do EaD. Sei que matemática não é fácil, ainda mais em situações desconfortáveis, (...) mesmo as aulas sendo à distância, gostei de lecionar e quero fazer isso de forma presencial, comparando as diferenças e semelhanças, pontos positivos e negativos das diferentes plataformas de ensino. Aos meus primeiros alunos, muito obrigado.

(Narrativa escrita pelo monitor dos círculos matemáticos da economia, 2020).

Do fragmento acima, o que nos chama a atenção (e esta escolha também faz parte da subjetividade com a qual analisamos toda a experiência) são as partes da narrativa que se voltam para os aspectos emocionais trazidos com o contexto no qual este projeto se insere: "trabalhei meu emocional e o emocional dos alunos, estávamos todos ansiosos"; "percebi uma certa falta de esperança em toda turma, não saberia julgar se por conta da pandemia e seus problemas ou em decorrência do EaD" e ainda "sei que matemática não é fácil, ainda mais em situações desconfortáveis".

Ainda utilizando da nossa subjetividade, após acompanharmos todo o processo desde a proposição do projeto, sua adaptação ao ensino remoto e sua execução no contexto da pandemia Covid-19, a análise interpretativa dos pontos destacados do fragmento evidencia que o convívio escolar, onde sejam compartilhadas expectativas emocionais, faz-se necessária em todos os ambientes escolares, presenciais ou digitais, como ponto de partida para a construção da aprendizagem. 


\section{Conclusões}

Os círculos matemáticos é um projeto de ensino, proposto para os alunos ingressantes do curso de Ciências Econômicas de uma universidade pública do estado de São Paulo para o ano letivo de 2020. Seu objetivo, além de proporcionar a melhoria do desempenho escolar e do raciocínio abstrato na resolução de problemas teóricos e aplicados ao dia a dia, consiste em promover um ambiente acadêmico de integração entre os alunos que estimule o estudo em grupo.

Neste artigo, fazemos um relato da experiência vivenciada nos círculos matemáticos no contexto da pandemia Covi-19. Para isto, descrevemos a metodologia das sessões dos círculos para o ensino remoto e os problemas trabalhados em cada sessão. Na sequência, fazemos uma análise da experiência utilizando os dados quantitativos extraídos de atividades digitais e do questionário de avaliação do projeto. Finalmente, coletamos a narrativa do monitor do projeto, feita a partir das experiências por ele vivenciadas.

De forma geral, dos dados coletados, avaliamos a experiência como uma rica oportunidade de aprendizagem para docentes e discentes. Podemos dizer que uma limitação dos resultados desta experiência seria o possível viés nas respostas do questionário proposto, já que as expectativas dos estudantes em participar deste projeto poderiam ensejar respostas equivocadas ao questionário. Além disso, este estudo é limitado aos dados em um curto período, tornando necessário o desenvolvimento de estudos longitudinais para avaliar o protagonismo, a participação e o aproveitamento dos ingressantes neste projeto.

No que se refere a esta experiência, observamos que ensino remoto emergencial provocado pela pandemia Covid-19 foi um desafio coletivo e por isso, acreditamos, que a exequibilidade do projeto está associada a dois pontos: mudanças epistemológicas em todos os sujeitos envolvidos e o convívio escolar possibilitado pelos encontros online, que criaram vínculos e mantiveram ativas as salas de aulas como espaço.

\section{Referências}

ABREU, Jamil Gomes; CAPELATO, Érika. Notas de Matemática Básica, Universidade Estadual Paulista "Júlio de Mesquita Filho" - UNESP, Araraquara, 2019.

ARRUDA, Eucidio Pimenta. Educação remota emergencial: elementos para políticas públicas na educação brasileira em tempos de Covid-19. EmRede-Revista de Educação a Distância, v. 7, n. 1, p. 257-275, 2020.

CRAWFORD, Joseph et al. COVID-19: 20 countries' higher education intra-period digital pedagogy responses. Journal of Applied Learning \& Teaching, v. 3, n. 1, p. 1-20, 2020.

DORICHENKO, Sergey. Um Círculo Matemático de Moscou: Problemas semana a semana. Instituto Nacional de Matemática Pura e Aplicada. Rio de Janeiro: IMPA, 2016.

PETERS, Michael A. et al. China's internationalized higher education during Covid-19: collective student autoethnography. Postdigital Science and Education, p. 1, 2020. 
SÃO PAULO. Decreto ${ }^{\circ}$ 64.862, de 13 março de 2020. Dispõe sobre a adoção, no âmbito da Administração Pública direta e indireta, de medidas temporárias e emergenciais de prevenção de contágio pelo COVID-19. Diário Oficial do Estado de São Paulo: v. 130, n. 51, 14 mar. 2020. Disponível em: https://www.al.sp.gov.br/norma/193314. Acesso em: 26 nov. 2020.

\section{Agradecimentos}

Agradecemos ao Programa Núcleo de Ensino da Unesp pelo financiamento do projeto, bem como ao monitor que atuou para a sua exequibilidade. 